\title{
Near-infrared line imaging of the starburst galaxies NGC 520, NGC 1614 and NGC 7714
}

\author{
J. K. Kotilainen ${ }^{1}$, J. Reunanen ${ }^{1}$, S. Laine ${ }^{2,3,4}$, and S. D. Ryder ${ }^{5}$ \\ 1 Tuorla Observatory, University of Turku, Väisäläntie 20, 21500 Piikkiö, Finland \\ 2 Department of Physical Sciences, University of Hertfordshire, College Lane, Hatfield, \\ Herts AL10 9AB, UK \\ 3 Department of Physics and Astronomy, University of Kentucky, Lexington, KY 40506-0055, USA \\ 4 Space Telescope Science Institute, 3700 San Martin Drive, Baltimore, MD 21218, USA \\ 5 Anglo-Australian Observatory, PO Box 296, Epping, NSW 1710, Australia
}

Received 9 August 2000 / Accepted 14 November 2000

\begin{abstract}
We present high spatial resolution $\left(\sim 00^{\prime \prime} 6\right)$ near-infrared broad-band $J H K$ images and $\operatorname{Br} \gamma 2.1661 \mu \mathrm{m}$ and $\mathrm{H}_{2} \quad 1-0 S(1) 2.122 \mu \mathrm{m}$ emission line images of the nuclear regions in the interacting starburst galaxies NGC 520, NGC 1614 and NGC 7714. The near-infrared emission line and radio morphologies are in general agreement, although there are differences in details. In NGC 1614, we detect a nuclear double structure in $\operatorname{Br} \gamma$, in agreement with the radio double structure. We derive average extinctions of $A_{K}=0.41$ and $A_{K}=0.18$ toward the nuclear regions of NGC 1614 and NGC 7714, respectively. For NGC 520, the extinction is much higher, $A_{K}=1.2-1.6$. The observed $\mathrm{H}_{2} / \mathrm{Br} \gamma$ ratios indicate that the main excitation mechanism of the molecular gas is fluorescence by intense UV radiation from clusters of hot young stars, while shock excitation can be ruled out. The starburst regions in all galaxies exhibit small $\mathrm{Br} \gamma$ equivalent widths. Assuming a constant star formation model, even with a lowered upper mass cutoff of $M_{\mathrm{u}}=30 M_{\odot}$, results in rather old ages (10-40 Myr), in disagreement with the clumpy near-infrared morphologies. We prefer a model of an instantaneous burst of star formation with $M_{\mathrm{u}}=100 M_{\odot}$, occurring $\sim 6-7 \mathrm{Myr}$ ago, in agreement with previous determinations and with the detection of W-R features in NGC 1614 and NGC 7714. Finally, we note a possible systematic difference in the amount of hot molecular gas between starburst and Seyfert galaxies.
\end{abstract}

Key words. galaxies: individual: NGC 520 - galaxies: individual: NGC 1614 - galaxies: individual: NGC 7714 galaxies: starburst - infrared: galaxies - stars: formation

\section{Introduction}

Interaction and merging play an important role in the evolution of galaxies. Interacting galaxies usually have strong infrared (IR) emission (e.g. Rieke et al. 1980; Telesco et al. 1988), and show a wide range of nuclear activity, including AGN, nuclear starbursts (SB), ultraluminous IR galaxies and post-SB activity. These phenomena probably all arise from the ability of interactions to transport gas into the galactic nuclei (e.g. Barnes \& Hernquist 1991) to fuel both AGN and SBs. In SB galaxies, the radiation output is dominated by star formation (SF), and SBs typically have SF rates of 5-50 $M_{\odot} \mathrm{yr}^{-1}$ within a region of $0.1-1 \mathrm{kpc}$ extent, much larger than that found in the Galactic center (e.g. Mezger et al. 1996) or in normal galaxies (e.g. Keel 1983), and they thus provide an extreme environment in which to study the SF process.

Send offprint requests to: J. K. Kotilainen, e-mail: jarkot@astro.utu.fi
Multiwavelength studies of interacting SB galaxies can provide insights into the connection between interactions, massive star clusters, and nuclear activity. The relatively unobscured near-IR (NIR) emission provides a better handle than optical emission to quantify the properties of SF in galaxies. In this paper we present high resolution NIR broad-band $J H K$ images and $\operatorname{Br} \gamma$ and $\mathrm{H}_{2}$ 1-0 S(1) emission line images of the circumnuclear regions of three interacting/merging SB galaxies, NGC 520, NGC 1614 and NGC 7714. Because of their proximity, brightness and reasonably large angular size, they provide excellent targets for these studies. $\mathrm{Br} \gamma$ originates from $\mathrm{H}$ II regions surrounding hot young $\mathrm{OB}$ star clusters, while $\mathrm{H}_{2}$ arises from hot molecular gas and traces the material available for SF.

In the remaining part of Sect. 1, we give brief introduction to the galaxies. In Sect. 2 we discuss the observations and data reduction. In Sect. 3 we discuss the morphology of the circumnuclear regions, determine the extinctions to the SF complexes, constrain their SF properties, 
stellar populations and SF history by comparison among the NIR tracers and with multiwavelength emission, and discuss the gas masses of the galaxies. Conclusions are drawn in Sect. 4.

\subsection{NGC 520}

NGC 520 (Arp 157, UGC 966, VV 231) at $v_{\text {sys }}=$ $2059 \mathrm{~km} \mathrm{~s}^{-1}\left(D=27.3 \mathrm{Mpc}, 1^{\prime \prime}=130 \mathrm{pc}\right.$, for $H_{0}=$ $75 \mathrm{Mpc}^{-1} \mathrm{~km} \mathrm{~s}^{-1}$ and $\left.q_{0}=0.5\right)$ is an IR-luminous peculiar pair of galaxies with a highly disturbed flattened morphology (e.g. Stanford \& Balcells 1990; Hibbard \& van Gorkom 1996). The inclination of NGC 520 is $\sim 66^{\circ}$ (Rownd \& Young 1999). NGC 520 is at an intermediate merger stage (Hibbard \& van Gorkom 1996), with the two nuclei embedded within a common stellar envelope. The primary nucleus (hereafter PN) is optically hidden behind a dust lane at $\mathrm{PA}=95^{\circ}$, while the second, optically bright nucleus (hereafter NWN) is situated $\sim 40^{\prime \prime}(5.3 \mathrm{kpc})$ to NW. A bright optical tail stretches $\sim 2$ ! $(22 \mathrm{kpc})$ to SE, bending sharply to E and connecting to a broad stellar plume.

The $\mathrm{PN}$ has $\sim 4.310^{9} M_{\odot}$ of molecular gas in a $\sim 7^{\prime \prime} \times 3^{\prime \prime}(920 \times 400 \mathrm{pc})$ scale rotating molecular gas disk at $\mathrm{PA}=95^{\circ}$ (Yun \& Hibbard 2000). While the CO emission is in good agreement with the $1.4 \mathrm{GHz}$ radio emission $\left(5^{\prime \prime} \times 2^{\prime \prime}=660 \times 260 \mathrm{pc}, \mathrm{PA}=93^{\circ} ;\right.$ Condon et al. 1990$)$, both avoid the $\mathrm{H} \alpha$ emission, which is dominated by plumes of ionized gas emerging up to a projected distance of $20^{\prime \prime}$ $(2.6 \mathrm{kpc})$ from the nucleus along $\mathrm{PA}=25^{\circ}$ (Hibbard \& van Gorkom 1996). These plumes probably represent a SB-driven bipolar outflow of ionized gas out of the nuclear SB region (Norman et al. 1996). While $\mathrm{H} \alpha$ emission regions exist within the $\mathrm{PN}$ region, the nuclear $\mathrm{SB}$ is completely obscured optically by dust within the nuclear gas disk (but becomes visible in $\operatorname{Br} \gamma$; Sect. 3.1.1).

NGC 520 is probably the result of an encounter $\sim 310^{8}$ yr ago between a gas-rich and a gas-poor galaxy (Stanford \& Barcells 1991). Both the S tail and the plume arose from the gas-poor progenitor and are currently disturbing the underlying gas distribution from the gas-rich disk (Hibbard \& van Gorkom 1996). The optical spectrum of the NWN is dominated by A stars, indicating that it is now in a post-SB phase (Stanford \& Balcells 1990; Bernlöhr 1993b), in agreement with the non-detection of $\mathrm{Br} \gamma$ emission (Sect. 3.1.1). Enhanced SF is currently detected only in the PN (e.g. Stanford 1991).

\subsection{NGC 1614}

NGC 1614 (Mrk 617, Arp 186, II $\mathrm{Zw} \mathrm{15)} \mathrm{is} \mathrm{an} \mathrm{IR-}$ luminous $\mathrm{SB}(\mathrm{s}) \mathrm{c}$ pec type $\mathrm{SB}$ galaxy at $v_{\text {sys }}=4723 \mathrm{~km} \mathrm{~s}^{-1}$ $\left(D=62.4 \mathrm{Mpc}, 1^{\prime \prime}=300 \mathrm{pc}\right)$. It has been extensively studied at optical (e.g. De Robertis \& Shaw 1988), NIR (e.g. Aitken et al. 1981; Forbes et al. 1992; Puxley \& Brand 1999) and radio (e.g. Condon et al. 1982) wavelengths. The inclination of NGC 1614 is $\sim 30^{\circ}$ (Rownd \& Young 1999).
The extended emission around NGC 1614 is highly asymmetric, suggesting recent tidal interaction with at least one other galaxy and that the galaxies are currently merging. The chaotic optical structure includes two roughly symmetrical inner spiral arms, a linear tail to the SW extending almost $1^{\prime}(18 \mathrm{kpc})$ from the nucleus and a large curving arc to the E/SE that reaches $\sim 33^{\prime \prime}$ (9.8 kpc) from the nucleus (e.g. Neff et al. 1990). Only a single peak is seen in the NIR continuum, suggesting that the two galaxies have already merged. However, two peaks (possibly double nuclei) are seen in the $\mathrm{Br} \gamma$ emission (Sect. 3.1.2) and in radio (Condon et al. 1982).

Armus et al. (1989) found a weak broad feature at $4660 \AA$ due to W-R stars in the nuclear spectrum, which was later confirmed by Vacca \& Conti (1992). Neff et al. (1990) made a multiwavelength study of NGC 1614 and concluded that its extreme IR luminosity results from vigorous nuclear SF induced by the interaction and merger of at least two galaxies. The molecular hydrogen mass of NGC 1614 is $M\left(\mathrm{H}_{2}\right)=1.110^{10} M_{\odot}$ (Sanders et al. 1991). There is an unresolved central CO source in NGC 1614 which emits $\sim 30 \%$ of the total CO flux (Scoville et al. 1989), implying a very high molecular gas concentration to the nucleus.

\subsection{NGC 7714}

NGC 7714 (Arp 284, VV 51, Mrk 538) is a nearby $\left(v_{\text {sys }}=\right.$ $\left.2808 \mathrm{kms}^{-1}, D=37.2 \mathrm{Mpc}, 1^{\prime \prime}=180 \mathrm{pc}\right)$ peculiar $\mathrm{SBb}$ type SB galaxy. It is very IR-luminous and has strong SB activity both in the nucleus and in circumnuclear regions (e.g. Gonzalez-Delgado et al. 1995, hereafter GD95; Garcia-Vargas et al. 1997). NGC 7714 and NGC 7715 (at $\sim 2^{\prime}(21 \mathrm{kpc})$ to the $\left.\mathrm{E} ; v_{\mathrm{sys}}=2770 \mathrm{~km} \mathrm{~s}^{-1}, D=36.7 \mathrm{Mpc}\right)$ form the Arp 284 system of interacting spirals, as is evident from the HI and optical bridge (Smith et al. 1997; Papaderos \& Fricke 1998) between them. The inclination of NGC 7714 is $\sim 42^{\circ}$ (Chapelon et al. 1999). A striking feature of NGC 7714 is an asymmetric circumnuclear ring of diameter $\sim 20^{\prime \prime} \times 40^{\prime \prime}(3.6 \times 7.2 \mathrm{kpc})$, with most of the emission located to the $\mathrm{E}$ of the nucleus. This ring is largely devoid of $\mathrm{H} \alpha$ (GD95) and HI emission (Smith et al. 1997), and has NIR colours similar to the old disk population (Bushouse \& Werner 1990). This structure is thus probably related to the dynamical perturbation of the disk rather than to previous SF. A weak He II $4686 \AA$ W$\mathrm{R}$ feature has been detected in NGC 7714 (GD95). The molecular gas mass of NGC 7714 is $M\left(\mathrm{H}_{2}\right)=2.110^{9} M_{\odot}$ (Sanders et al. 1991).

While NGC 7714 shows strong SB activity, NGC 7715 has no optical emission lines and is in a post-SB phase (Bernlöhr 1993a). There are three main circumnuclear HII regions in NGC 7714, labelled A, B and C by GD95, and located in the bulge/disk of the galaxy at $5^{\prime \prime}(900 \mathrm{pc}) \mathrm{E}$, $12^{\prime \prime}(2.1 \mathrm{kpc}) \mathrm{NW}$ and $14^{\prime \prime}(2.5 \mathrm{kpc}) \mathrm{SW}$ from the nucleus, respectively. Region A is detected by us as $\mathrm{Br} \gamma$ region 4. In the deconvolved $\mathrm{H} \alpha$ image of GD95, the nuclear SB region 
breaks furthermore into the nucleus and an extranuclear component, which we identify as $\operatorname{Br} \gamma$ region 2.

\section{Observations and data reduction}

The observations of the $\operatorname{Br} \gamma 2.1661 \mu \mathrm{m}$ and $\mathrm{H}_{2}$ 1-0 S(1) $2.121 \mu \mathrm{m}$ emission lines and the $J H K$ bands were carried out in September 1998 with the 3.8 m United Kingdom Infrared Telescope (UKIRT) on Mauna Kea, Hawaii, under FWHM 0.'6-0.'7 seeing. We used the $256 \times 256 \mathrm{px}$ IRCAM3 camera, with pixel size $0.281 \mathrm{px}^{-1}$ and field of view $\sim 70^{\prime \prime} \times 70^{\prime \prime}$. For the emission line observations, we used cooled $(T=77 \mathrm{~K})$ narrow-band filters and a FabryPerot $(\mathrm{F}-\mathrm{P})$ etalon with spectral resolution $\sim 400 \mathrm{~km} \mathrm{~s}^{-1}$ and equivalent width $(E W) 0.0038 \mu \mathrm{m}$. For the emission lines, sequences of $1 \mathrm{~min}$ jittered observations in a five point grid were made at the on-line wavelength and at the nearby blue and red continuum. For the $J H K$-bands, $30 \mathrm{~s}$ integrations were made both centered on the galaxy and on the adjacent sky. The total integration times were 40, 10 and $30 \mathrm{~min}$ in $\mathrm{Br} \gamma, 23,10$ and $30 \mathrm{~min}$ in $\mathrm{H}_{2}$ and 2.5-7.5 min in the $J H K$-bands, for NGC 520, NGC 1614 and NGC 7714, respectively.

The line images were linearized, dark-subtracted, flatfielded and sky-subtracted using $\mathrm{IRAF}^{1}$. These images were aligned to within a small fraction of a pixel using field stars or the nucleus as reference, and merged into one on-line image and two continuum images. The continuum images were scaled, combined, and subtracted from the on-line images, and the final line images were flux calibrated against spectral type A standard stars.

The sensitivity of the F-P depends on both the lineof-sight (l.o.s.) velocity of the galaxy and the shift in the transmitted wavelength of the $\mathrm{F}-\mathrm{P}$ over the field of view. These effects were corrected for by dividing the measured fluxes by an inverse Airy function (e.g. Bland-Hawthorn 1995). For NGC 520 and NGC 7714, we used the l.o.s. $\mathrm{H} \alpha$ velocity fields by Bernlöhr $\left(1993 \mathrm{~b} ; \mathrm{PA}=120^{\circ}\right.$ and $\left.\mathrm{PA}=145^{\circ}\right)$ and GD95 $\left(\mathrm{PA}=110^{\circ}\right.$ and $\left.\mathrm{PA}=216^{\circ}\right)$, respectively. For NGC 1614, we are not aware of a published velocity field, and we used the $\mathrm{H} \alpha \mathrm{N}-\mathrm{S}$ rotation curve by De Robertis \& Shaw (1988) assuming axisymmetry. In the nuclei of all galaxies, we assumed the l.o.s. rotational velocity to be $0 \mathrm{~km} \mathrm{~s}^{-1}$. The emission line images have not been corrected for by the inverse Airy function, because noise would then depend on the position in the image. However, the observed emission line fluxes have been corrected for. To enhance $S / N$ and to detect faint structures, all images were slightly smoothed to $\leq 1^{\prime \prime}$ resolution.

Photometry in all bands was performed at the location of the detected $\mathrm{Br} \gamma$ emission regions. The aperture used for each region was selected to include as much emission as possible, while avoiding overlap with neighbouring

\footnotetext{
${ }^{1}$ IRAF is distributed by the National Optical Astronomy Observatories, which are operated by the Association of Universities for Research in Astronomy, Inc., under cooperative agreement with the National Science Foundation.
}

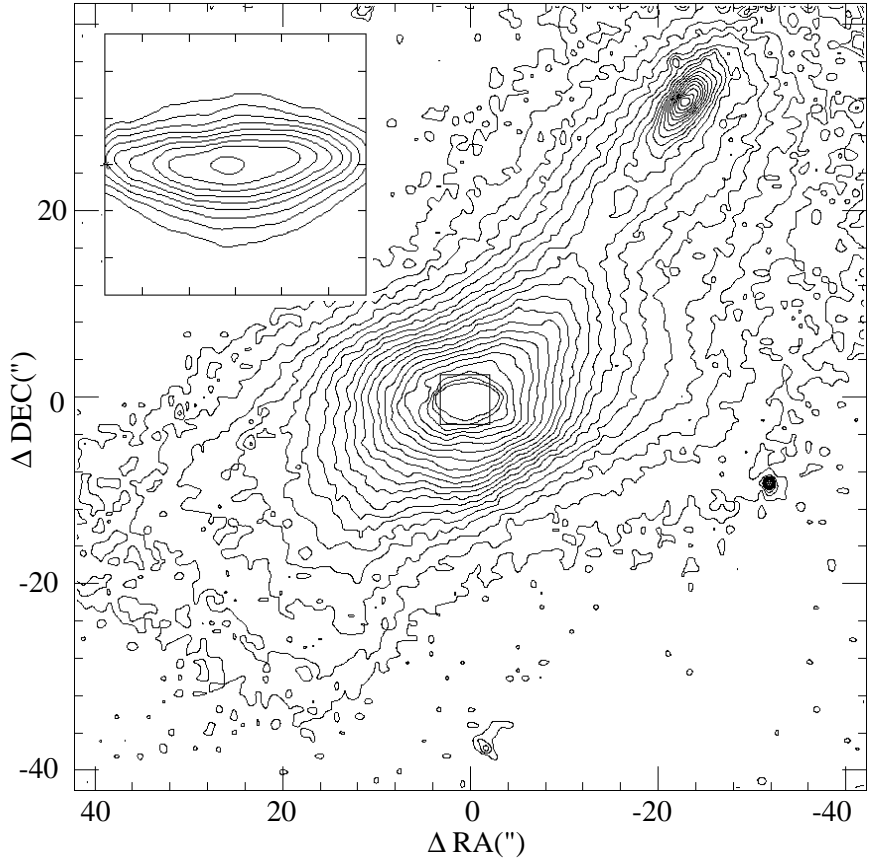

Fig. 1. The $K$-band image of NGC 520 with logarithmic intervals. The inset shows the central $6^{\prime \prime} \times 6^{\prime \prime}$ of NGC $520 \mathrm{PN}$. In this and all subsequent figures, north is up and east to the left

regions. The smallest distance between the nearest emission regions in these galaxies is $\sim 0^{\prime \prime} .8$, which is larger than the seeing during the observations $\left(\sim 00^{\prime \prime} 6\right)$. The observed fluxes were corrected for Galactic extinction and redshift (K-correction). We estimate a photometric accuracy in the $J H K$ magnitudes $\sim 0.03 \mathrm{mag}$, in the $J H K$ colours $\sim 0.05 \mathrm{mag}$, and in the emission line fluxes $\sim 10 \%$.

\section{Results and discussion}

\subsection{Morphology}

\subsubsection{NGC 520}

The $K$-band image of NGC 520 is shown in Fig. 1 (see also e.g. Stanford \& Balcells 1990). In the $J$-band (not shown), absorption of the stellar light distribution along the PN dust lane is noticeable (as in the optical). However, a stellar bulge, bisected by the dust lane, begins to become evident in the PN. The overall appearance of the $\mathrm{PN}$ is that of an edge-on stellar disk. In the $J$-band, the NWN stellar bulge still dominates the emission from the NGC 520 system. On the other hand, in the $K$-band, the dust lane no longer significantly obscures the underlying stellar light in the PN. The stellar bulge is seen clearly at the position of the center of the optical dust lane. The $\mathrm{PN}$ is elongated in roughly $\mathrm{E}-\mathrm{W}$ direction, while the outer bulge region appears more spherical. The position of the $K$-band $\mathrm{PN}$ coincides with the radio continuum and $\mathrm{CO}$ peaks. The NWN is $\sim 4$ times fainter than the PN in the $K$-band, indicating that it is the less massive of the two bulges (stellar masses of the old population are $910^{9} M_{\odot}$ 


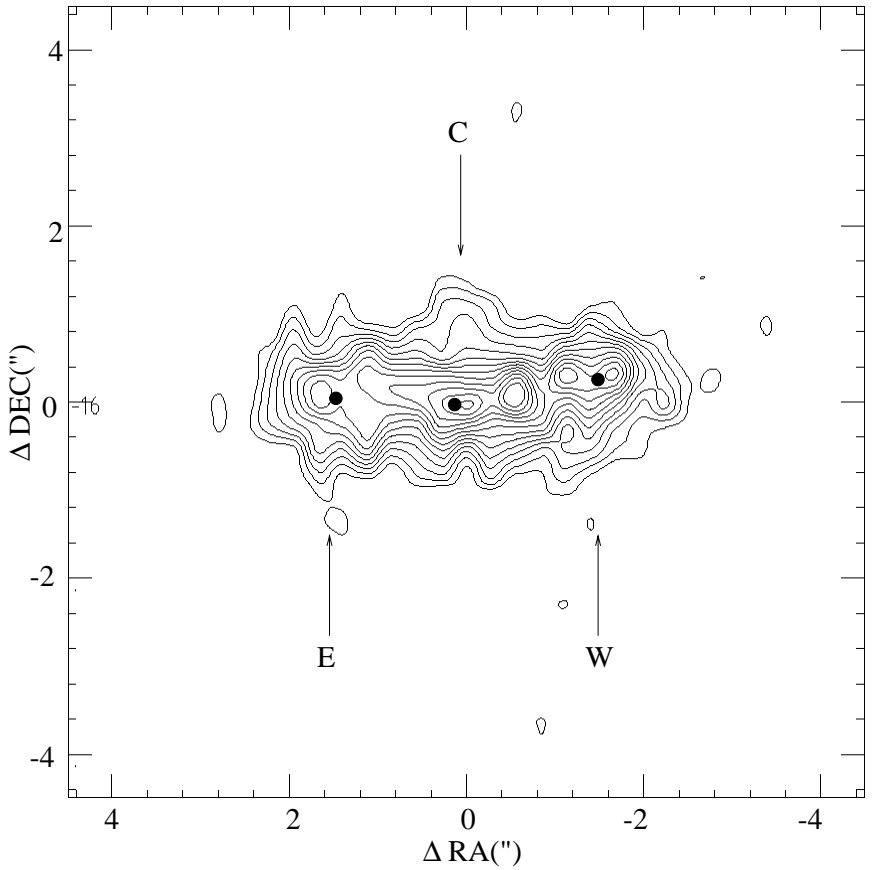

Fig. 2. The $\mathrm{Br} \gamma$ emission of NGC $520 \mathrm{PN}$. The lowest contour is at $19 \%$ level of the maximum and corresponds to $3 \sigma$. The other contours are at $1 \sigma$ intervals. The maximum surface brightness is $2.410^{-15} \mathrm{erg} \mathrm{s}^{-1} \mathrm{~cm}^{-2} \operatorname{arcsec}^{-2}$. The black dots correspond to the three $\operatorname{Br} \gamma$ emission regions studied in this work

and $210^{9} M_{\odot}$ for the PN and NWN, respectively; Stanford \& Balcells 1990).

We have detected $\operatorname{Br} \gamma$ emission at higher than $3 \sigma$ level in the central $\sim 5^{\prime \prime} \times 3^{\prime \prime}(660 \times 400 \mathrm{pc})$ region of NGC $520 \mathrm{PN}$ (Fig. 2). This emission describes a flattened morphology and breaks up into several components. We have selected three emission regions for further study, labelled $\mathrm{E}$ (east), $\mathrm{C}$ (center) and $\mathrm{W}$ (west). The total Br $\gamma$ flux from NGC $520 \mathrm{PN}, \sim 1.210^{-14} \mathrm{erg} \mathrm{s}^{-1} \mathrm{~cm}^{-2}$ is in reasonable agreement with previous values, e.g. $1.910^{-14} \mathrm{erg} \mathrm{s}^{-1} \mathrm{~cm}^{-2}$ in a $6^{\prime \prime} \times 8^{\prime \prime}$ aperture (Stanford 1991).

There is very good spatial correspondence between the brightest regions in the $\operatorname{Br} \gamma$ and the radio emission. At $5 \mathrm{GHz}$, NGC $520 \mathrm{PN}$ is an extended linear triple radio source with dimensions $\sim 6^{\prime \prime} \times 1^{\prime \prime} .8(790 \times 240 \mathrm{pc})$ at $\mathrm{PA}=93^{\circ}$ (Condon et al. 1982). At $15 \mathrm{GHz}$, the emission is resolved into five components at $\mathrm{PA} \sim 90^{\circ}$ (Carral et al. 1991). Because the non-thermal radio emission is believed to arise in supernova (SN) remnants and the thermal radio emission to be reradiated UV emission from OB stars, this correspondence is not surprising.

The correspondence between the $\mathrm{H}_{2}$ (Fig. 3) and the $\mathrm{Br} \gamma$ emission in the $\mathrm{PN}$ is reasonably good. Both emission lines define an elongated structure in roughly E-W direction. The $\mathrm{H}_{2}$ emission is more extended, with maximum dimensions of $\sim 8^{\prime \prime} \times 4^{\prime \prime}(1.1 \times 0.5 \mathrm{kpc})$. There is, however, no clear correlation between the $\mathrm{Br} \gamma$ and $\mathrm{H}_{2}$ peaks. The total $\mathrm{H}_{2}$ flux from NGC $520 \mathrm{PN}, \sim 0.710^{-14} \mathrm{erg} \mathrm{s}^{-1} \mathrm{~cm}^{-2}$

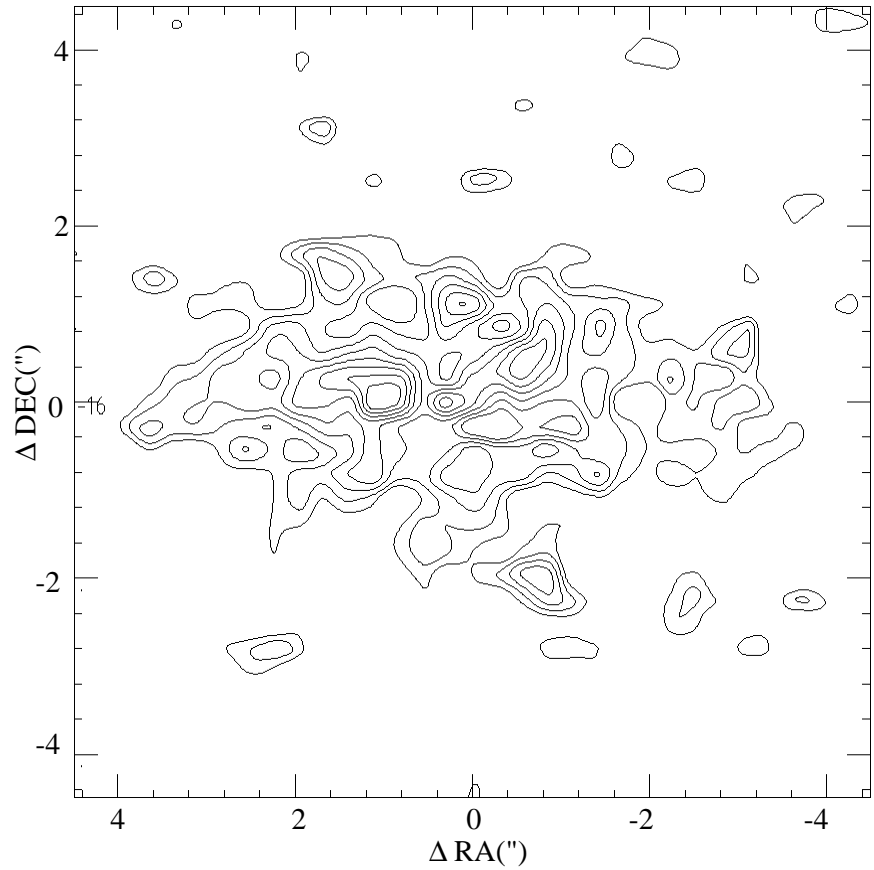

Fig. 3. The $\mathrm{H}_{2}$ emission of NGC $520 \mathrm{PN}$. The lowest contour is at $28 \%$ level of the maximum and corresponds to $3 \sigma$. The other contours are at $1 \sigma$ intervals. The maximum surface brightness is $0.9310^{-15} \mathrm{erg} \mathrm{s}^{-1} \mathrm{~cm}^{-2} \operatorname{arcsec}^{-2}$

is smaller than the flux $1.910^{-14} \mathrm{erg} \mathrm{s}^{-1} \mathrm{~cm}^{-2}$ in a $6^{\prime \prime}$ $\times 8^{\prime \prime}$ aperture (Stanford 1991). However, as the spectrum of Stanford (1991) covers only a few pixels, suggesting the possibility of contamination from alignment and/or bad pixels, this comparison has to be approached with caution.

The $H-K$ colour map of NGC $520 \mathrm{PN}$ is shown in Fig. 4. The nuclear $H-K$ colour is very red, corresponding to extinction of $A_{K} \sim 1.5$. Note that the reddest peak is displaced from the $K$-band nucleus by $\sim 0^{\prime \prime} .3(40 \mathrm{pc}$ ) to the E. The size and the PA of the red region is similar to that of the $\mathrm{Br} \gamma$ emission. Outward the extinction quickly becomes much less severe. There is no spatial correlation between the $\mathrm{H} \alpha$ (Bernlöhr 1993b) and the $\mathrm{Br} \gamma$ emission in the $\mathrm{PN}$, again indicating that the effect of extinction is severe in NGC 520 (see also Sect. 3.2.1).

\subsubsection{NGC 1614}

The $K$-band image of NGC 1614 (Fig. 5) clearly shows the inner spiral arms, but is not deep enough to show the SW tail. However, the SF regions SW and W of the nucleus are detected, although they remained undetected in the NIR image of Neff et al. (1990). At very faint levels, we also see evidence for the beginning of the optical curved arc to the $\mathrm{E}$ of the nucleus.

We have detected $\operatorname{Br} \gamma$ emission at higher than $3 \sigma$ level in the central $\sim 3^{\prime \prime} .5 \times 33^{\prime \prime} .5(1.0 \times 1.0 \mathrm{kpc})$ region of NGC 1614 (Fig. 6). Intriguingly, this emission is resolved into two main components (SE and NW) straddling the nucleus and separated by $\sim 0^{\prime \prime} 7(210 \mathrm{pc})$ in roughly 


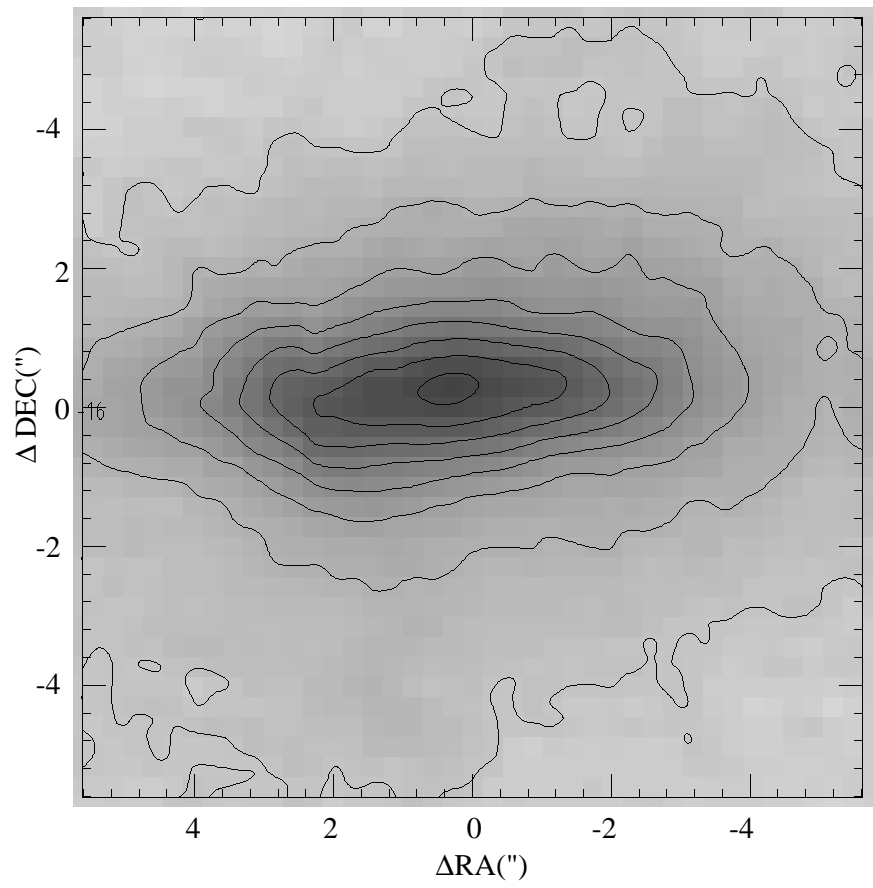

Fig. 4. The $H-K$ colour map of NGC 520 in greyscale and contours. The highest contour corresponds to $H-K=1.3$ and the other contours are at $H-K=0.15$ intervals

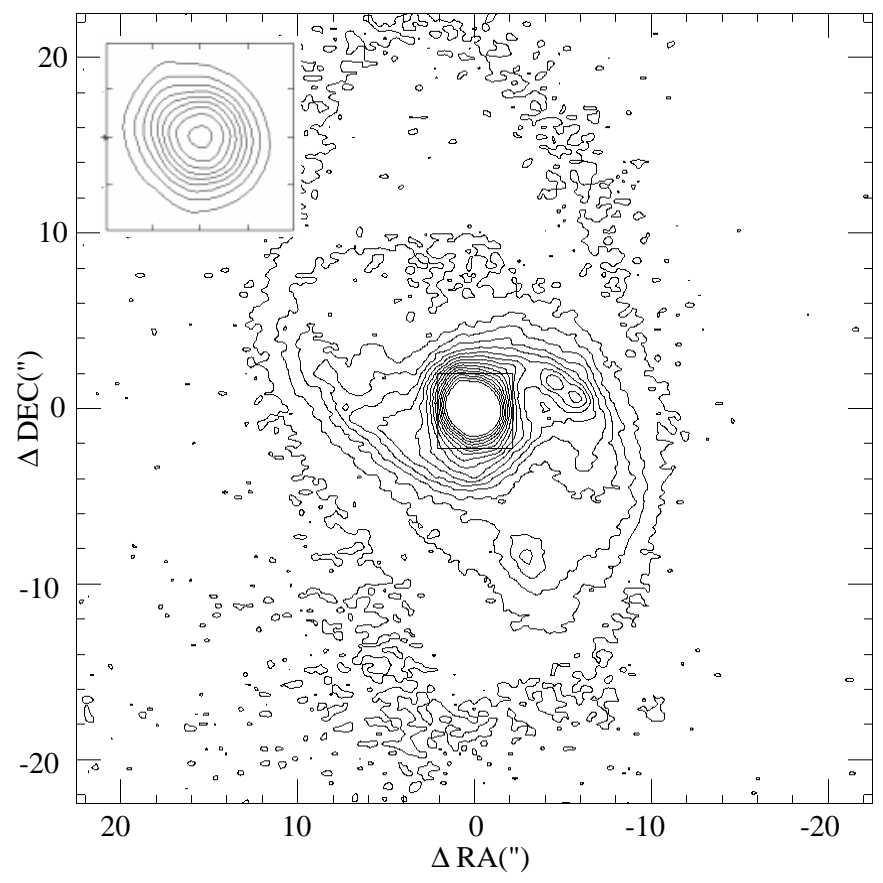

Fig. 5. The $K$-band image of NGC 1614 with logarithmic intervals. The inset shows the central $4^{\prime \prime} \times 4^{\prime \prime}$ region

SE-NW direction. The total $\mathrm{Br} \gamma$ flux from NGC 1614, $\sim 2.410^{-14} \mathrm{erg} \mathrm{s}^{-1} \mathrm{~cm}^{-2}$ is somewhat smaller than previously reported values, e.g. $7.210^{-14} \mathrm{erg} \mathrm{s}^{-1} \mathrm{~cm}^{-2}$ in a $7^{\prime \prime}$ aperture (Ho et al. 1990), 3.6 $10^{-14} \mathrm{erg} \mathrm{s}^{-1} \mathrm{~cm}^{-2}$ in a $3^{\prime \prime} \times$ $4^{\prime \prime}$ aperture (Neff et al. 1990) and $6.010^{-14} \mathrm{erg} \mathrm{s}^{-1} \mathrm{~cm}^{-2}$ (Puxley \& Brand 1999). This difference suggests that a part of the more extended $\operatorname{Br} \gamma$ emission may have

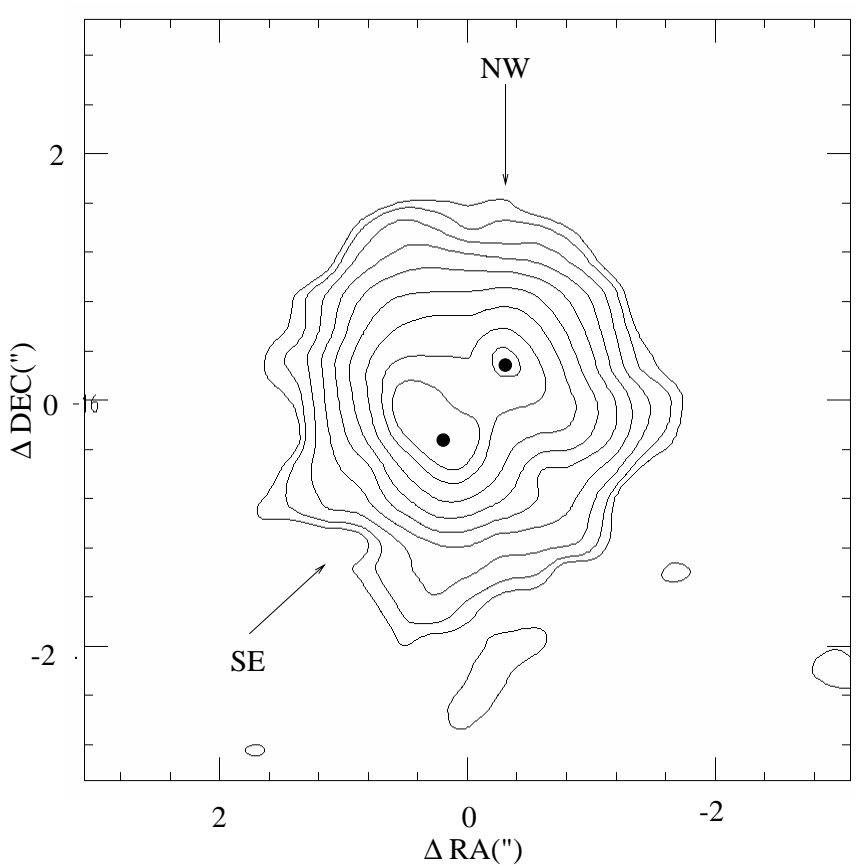

Fig. 6. The $\operatorname{Br} \gamma$ emission of NGC 1614. The lowest contour is at $7.1 \%$ level of the maximum and corresponds to $3 \sigma$. The other contours are at 9.5, 14, 21, 31, 43, 57, 74 and $86 \%$ of the maximum. The maximum surface brightness is $7.510^{-15} \mathrm{erg} \mathrm{s}^{-1} \mathrm{~cm}^{-2} \operatorname{arcsec}^{-2}$. The black dots correspond to the two $\mathrm{Br} \gamma$ emission regions studied in this work

remained undetected in our observation, due to the relatively short integration time.

This is the first time that the double structure is seen in the NIR. Note that it is not visible in our (Fig. 5) or published $K$-band images (e.g. Neff et al. 1990; Forbes et al. 1992), due to poorer spatial resolution and heavy foreground extinction. However, their existence was suggested by Puxley \& Brand (1999) who detected two components of roughly similar brightness in a velocityresolved Br $\gamma$ spectrum of the nucleus of NGC 1614.

The $5 \mathrm{GHz}$ radio emission of NGC 1614 (Neff et al. 1990) is in good agreement with the $\mathrm{Br} \gamma$ emission, showing two similar brightness maxima NW and SE of the nucleus, separated by $\sim 1^{\prime \prime} 2(360 \mathrm{pc})$. The total extent of emission is $\sim 3^{\prime \prime} \times 3^{\prime \prime}(890 \times 890 \mathrm{pc})$ for both radio and $\operatorname{Br} \gamma$. The center of NGC 1614 is resolved into two nuclei of similar brightness also at mid-IR $12.5 \mu \mathrm{m}$ wavelength (Keto et al. $1992)$, oriented $\sim \mathrm{E}-\mathrm{W}$ and separated by $\sim 0^{\prime \prime} 8$ (240 pc). An arm reaches $\mathrm{N}$ of the $\mathrm{W}$ component and then arches over to the E. This mid-IR structure resembles the double source observed in the $5 \mathrm{GHz}$ radio emission and in $\mathrm{Br} \gamma$, although the orientation is slightly different.

The double peaks in NGC 1614 are probably analogous to the double nuclei observed in many other IR-luminous galaxies. The separation of the nuclei in NGC 1614, $\sim 0^{\prime \prime}$.7 (210 pc) is slightly smaller than e.g. in Arp 220 (330 pc; Graham et al. 1990), and considerably less than the separations of $2-6 \mathrm{kpc}$ observed in several ultraluminous IR galaxies (Carico et al. 1990). On the other 


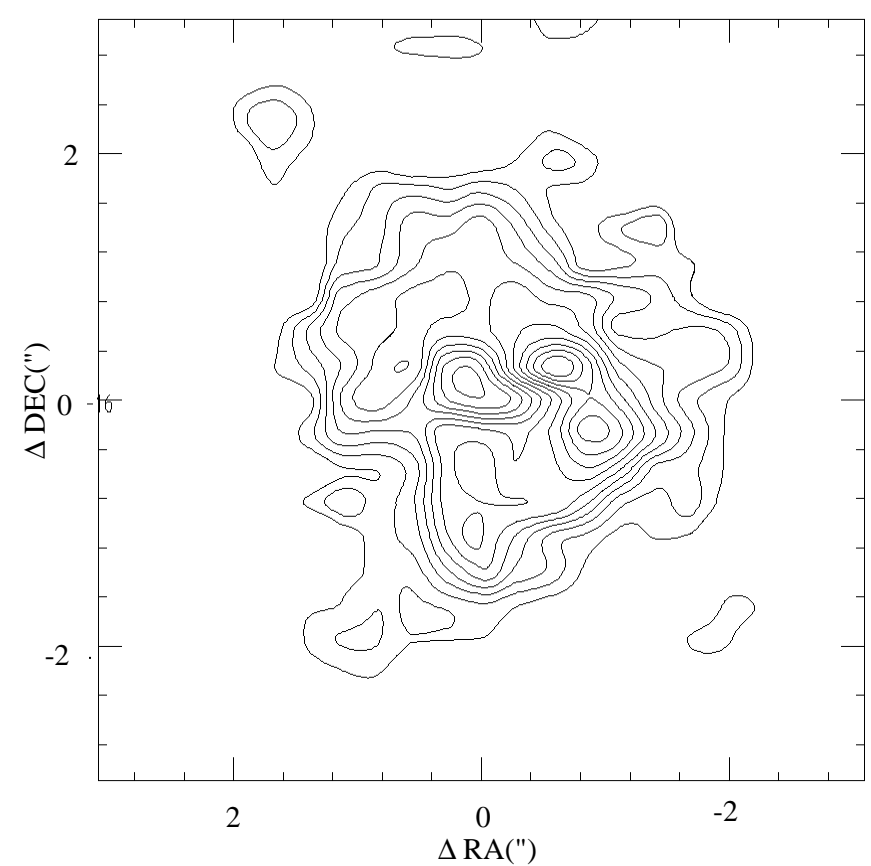

Fig. 7. The $\mathrm{H}_{2}$ emission of NGC 1614. The lowest contour is at $22 \%$ level of the maximum and corresponds to $3 \sigma$. The other contours are at $1 \sigma$ intervals. The maximum surface brightness is $1.810^{-15} \mathrm{erg} \mathrm{s}^{-1} \mathrm{~cm}^{-2} \operatorname{arcsec}^{-2}$

hand, NGC 1614 shows the structural characteristics of a collision with small impact parameter. The small separation of the nuclei and the high central molecular gas density (Scoville et al. 1989) should be inducive for AGN-type activity. However, multiwavelength information (Neff et al. 1990) strongly suggests that the nuclear emission of NGC 1614 is dominated by SB activity.

The correspondence between the $\mathrm{H}_{2}$ (Fig. 7) and the $\mathrm{Br} \gamma$ emission in NGC 1614 is reasonably good. The $\mathrm{H}_{2}$ emission is slightly more extended than $\mathrm{Br} \gamma$, with maximum dimensions of $\sim 4^{\prime \prime} \times 4^{\prime \prime}(1.2 \times 1.2 \mathrm{kpc})$. The $\mathrm{H}_{2}$ emission is resolved into several peaks, but there is no clear correlation with the $\mathrm{Br} \gamma$ peaks. The $H-K$ colour map of NGC 1614 is shown in Fig. 8. The central region is much redder than the rest of the galaxy. The $H-K$ map resolves this region of high extinction into a circumnuclear ring-like structure, with the $\operatorname{Br} \gamma$ maxima situated at the inner edges of this ring.

\subsubsection{NGC 7714}

The $K$-band image of NGC 7714 is shown in Fig. 9. In addition to the strong nuclear emission and the inner spiral arms, there is some evidence for the detection of the $\sim 20^{\prime \prime} \times 40^{\prime \prime}(3.6 \times 7.2 \mathrm{kpc})$ scale stellar ring to the $\mathrm{E}$ of the nucleus. We have detected $\operatorname{Br} \gamma$ emission at higher than $3 \sigma$ level in the central $\sim 6^{\prime \prime} \times 7^{\prime \prime}(1.1 \times 1.3 \mathrm{kpc})$ region of NGC 7714 (Fig. 10). This emission is not symmetric, but consists of the nuclear emission and a chain of $\operatorname{Br} \gamma$ regions (2, 3 and 4) toward $\mathrm{SE}$ of the nucleus. The strongest $\operatorname{Br} \gamma$ region (1) is not coincident with the

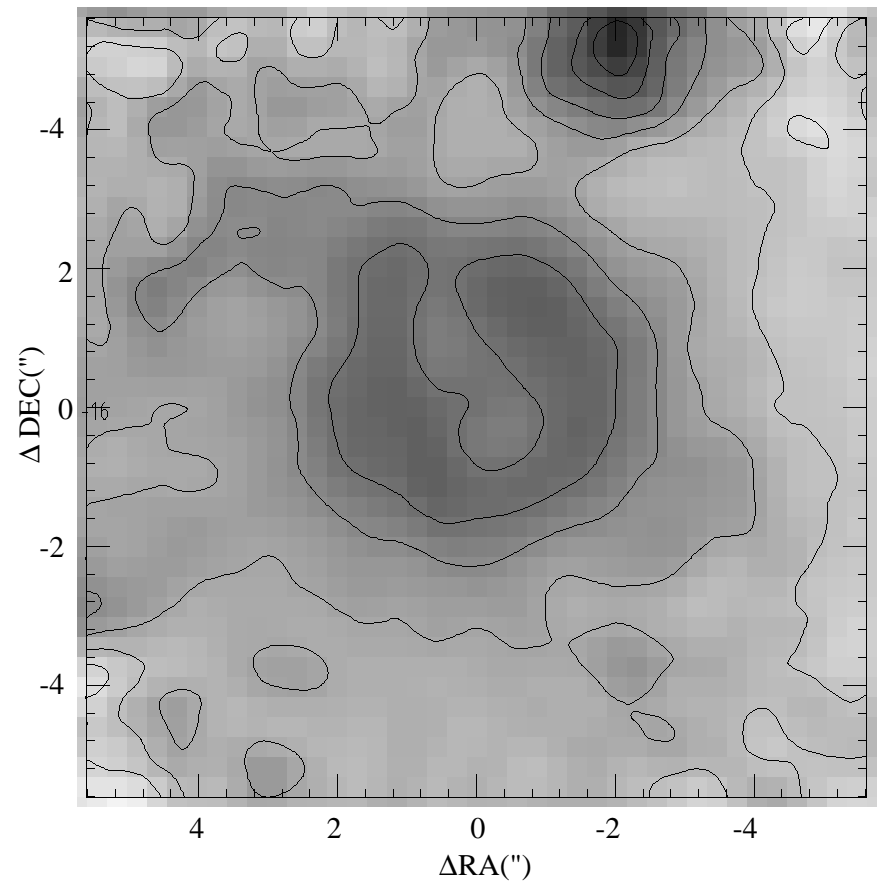

Fig. 8. The $H-K$ colour map of NGC 1614. The highest contour corresponds to $H-K=0.64$ and the other contours are at $H-K=0.08$ intervals

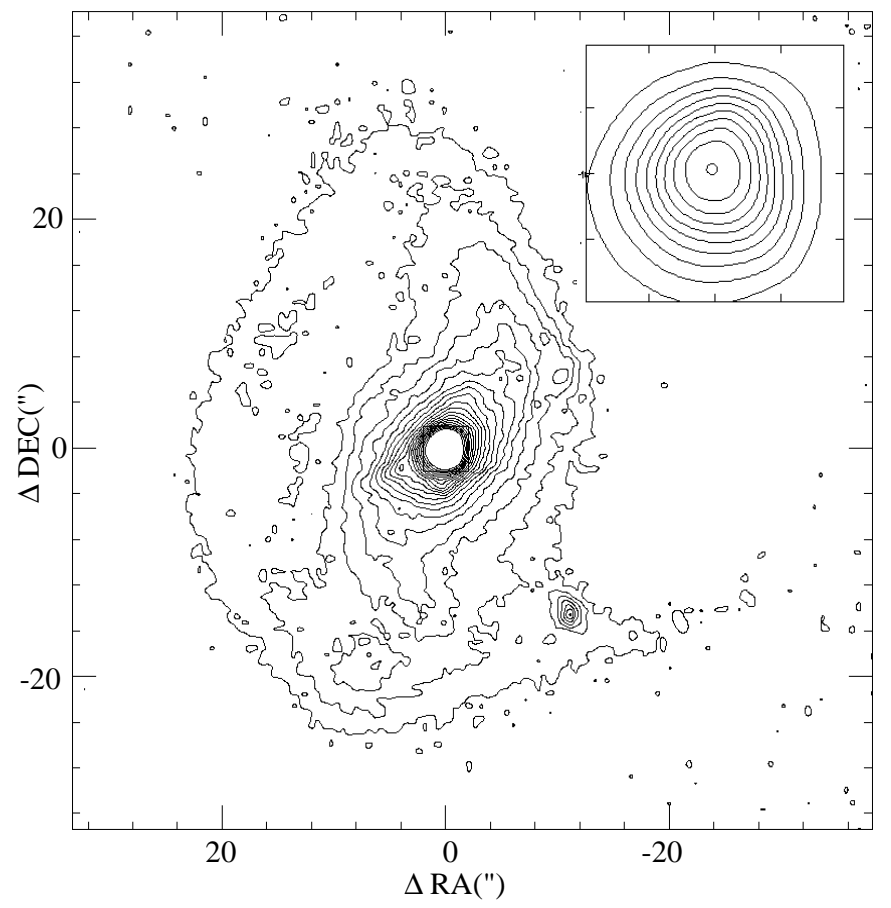

Fig. 9. The $K$-band image of NGC 7714 with logarithmic intervals. The inset shows the central $4^{\prime \prime} \times 4^{\prime \prime}$ region. There is a foreground star toward SW of the nucleus

nucleus, but is situated $\sim 0$ "! $8(140 \mathrm{pc})$ to SW. Its photometry was performed in a rectangular aperture to avoid the extended emission $\mathrm{N}$ of the nucleus and the nuclear emission. Interestingly, there is also indication of a fainter peak $\sim 0$ !' 4 (70 pc) to NE of the nucleus, with spiral-like emission connecting the two peaks. If real, this would add 


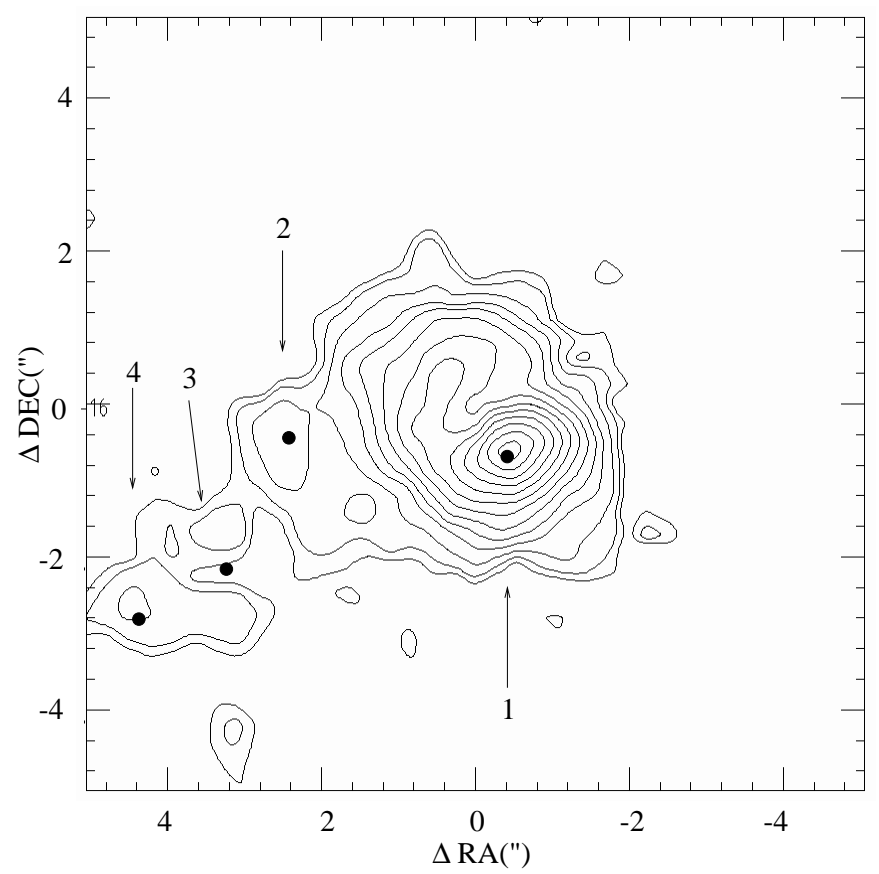

Fig. 10. The $\operatorname{Br} \gamma$ emission of NGC 7714. The lowest contour is at $4.1 \%$ level of the maximum and corresponds to $3 \sigma$. The other contours are at $5.4,8.2,12,16,22,29,46,57,69,83$ and $98 \%$ of the maximum. The maximum surface brightness is $7.010^{-15} \mathrm{erg} \mathrm{s}^{-1} \mathrm{~cm}^{-2} \operatorname{arcsec}^{-2}$. The black dots correspond to the four $\operatorname{Br} \gamma$ emission regions studied in this work

NGC 7714 to the increasing number of active galaxies with nuclear SF rings. The total Br $\gamma$ flux of NGC 7714, $\sim 2.710^{-14} \mathrm{erg} \mathrm{s}^{-1} \mathrm{~cm}^{-2}$ is slightly smaller than previously reported fluxes, e.g. $4.710^{-14}$ in a $7^{\prime \prime}$ aperture (Ho et al. 1990).

The $5 \mathrm{GHz}$ radio emission of NGC 7714 (Condon et al. 1982) shows two main components at $\mathrm{PA} \sim 45^{\circ}$ separated by $\sim 1$.'2 (210 pc), with the SW component being stronger. This morphology is in good agreement with the $\operatorname{Br} \gamma$ emission. There is a rather good correspondence between the $\mathrm{H}_{2}$ (Fig. 11) and the $\mathrm{Br} \gamma$ emission in NGC 7714. The maximum dimension of the detected $\mathrm{H}_{2}$ emission is $\sim 6^{\prime \prime} \times 8^{\prime \prime}$ $(1.1 \times 1.4 \mathrm{kpc})$, and it is resolved into several peaks, in reasonable agreement with $\mathrm{Br} \gamma$.

The nuclear SB extends to $\sim 16^{\prime \prime} \times 12^{\prime \prime}(2.9 \times 2.1 \mathrm{kpc})$ at $\mathrm{PA} \sim 110^{\circ}$ in the $\mathrm{H} \alpha$ emission (GD95). This extended nuclear SB is surrounded by a circumnuclear ring of small $\mathrm{H}$ II regions at $\sim 8^{\prime \prime}(1.4 \mathrm{kpc})$ radius and two giant $\mathrm{H}$ II regions at $12^{\prime \prime}(2.1 \mathrm{kpc})$ and $22^{\prime \prime}(3.9 \mathrm{kpc})$ from the nucleus. There is a good correlation between the $\mathrm{H} \alpha$ regions of GD95 and the $\operatorname{Br} \gamma$ emission, assuming that the nuclear $\mathrm{H} \alpha$ emission is coincident with our region 1 . Region 4 also has a counterpart in the $\mathrm{H} \alpha$ image to within 0 !' 1 . We have not detected $\mathrm{Br} \gamma$ emission further out from the nucleus, as opposed to the $\mathrm{H} \alpha$ imaging of GD95, suggesting a low value of extinction outside the nuclear region. The $H-K$ colour map of NGC 7714 is shown in Fig. 12. The effects of extinction are the least severe of all the galaxies

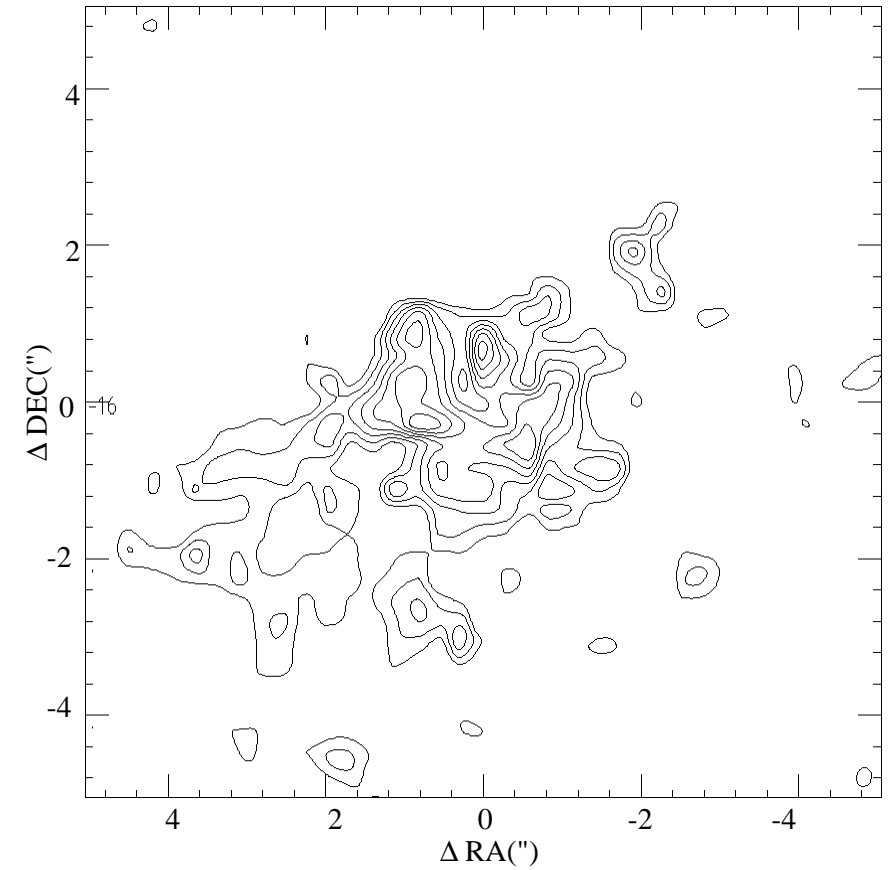

Fig. 11. The $\mathrm{H}_{2}$ emission of NGC 7714. The lowest contour is at $31 \%$ level of the maximum and corresponds to $3 \sigma$. The other contours are at $1 \sigma$ intervals. The maximum surface brightness is $5.910^{-16} \mathrm{erg} \mathrm{s}^{-1} \mathrm{~cm}^{-2} \operatorname{arcsec}^{-2}$

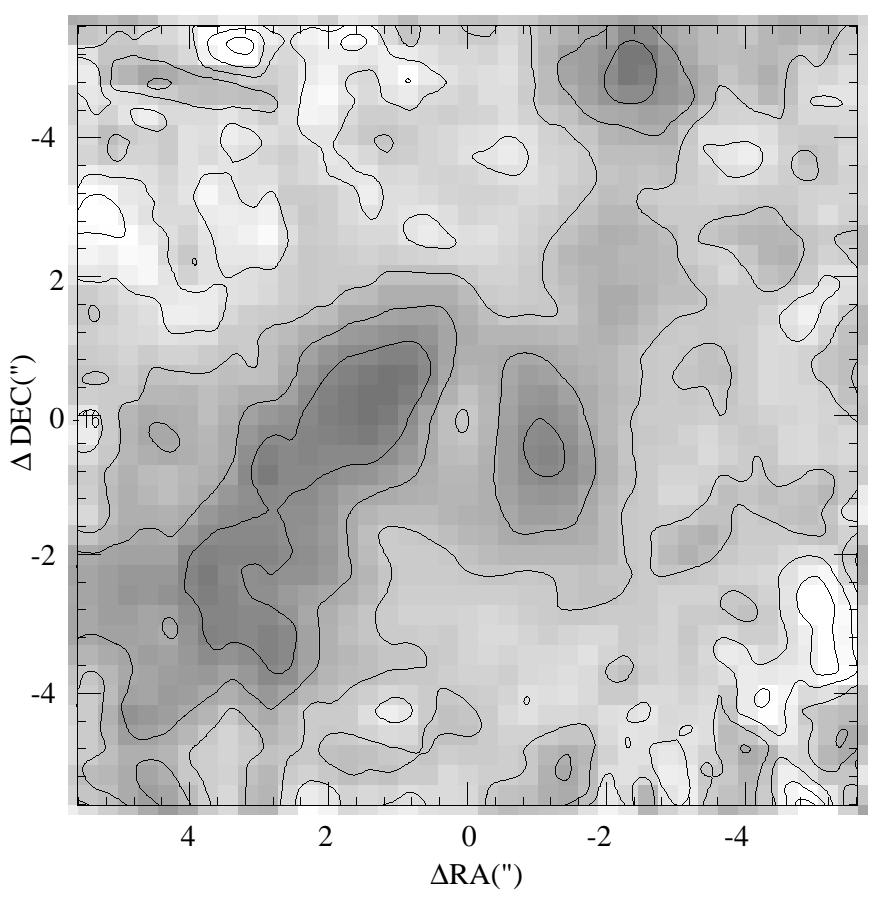

Fig. 12. The $H-K$ colour map of NGC 7714 . The highest contour corresponds to $H-K=0.35$ and the other contours are at $H-K=0.05$ intervals

studied here. The reddest regions in the $H-K$ map are in good agreement with the morphology of the $\operatorname{Br} \gamma$ emission (Fig. 10).

\subsection{Extinction}

We have estimated the extinction towards the ionized sources in NGC 1614 and NGC 7714 from published 
recombination line fluxes (Aitken et al. 1981; Taniguchi et al. 1988; Armus et al. 1989; Puxley \& Brand 1994; Storchi-Bergmann et al. 1995; Calzetti 1997). We have assumed standard interstellar dust properties, case B recombination, and the interstellar extinction law $A_{V} \propto \lambda^{-1.85}$ (Landini et al. 1984), and calculated the average integrated extinction applicable to the central region of both galaxies. For NGC 520 PN, this method cannot be used since it is optically obscured, and the extinction was assumed to be that calculated from the continuum colours (see below).

Calzetti et al. (1996) found that the geometry of the dust obscuring the ionized gas in a sample of 13 SBs can be well described by either homogeneous or clumpy foreground distribution and that the reddening is compatible with a Galactic-type extinction curve. Thus, most of the dust is located around but outside the SB region. We are unable to justify either a homogeneous or a clumpy foreground dust screen based on our data. For simplicity and for easy comparison with previous results, we have assumed the case of a homogeneous foreground dust screen, but for caveats, see Kotilainen et al. (2000). Note that the exact value of extinction does not affect the $E W \mathrm{~s}$ of the emission lines, if the differential extinction between the lines and the continuum is small (e.g. Calzetti 1997).

The extinction towards the continuum sources was estimated by comparing the observed $J H K$ colours of the emission regions to those of normal unobscured spiral galaxies (Glass \& Moorwood 1985). This method, however, may be biased toward low extinction regions, as the most heavily reddened regions may not be detectable in the $J H K$ images. Also, the intrinsic colours of the galaxies may be bluer than in normal galaxies, leading to an underestimation of the continuum extinction.

\subsubsection{NGC 520}

We used the continuum method for NGC 520, since $\mathrm{H} \alpha$ emission is completely obscured in the PN (Hibbard \& van Gorkom 1996). We derive for the emission regions in NGC $520 \mathrm{PN}$ very large extinctions of $A_{K}=1.2-1.6$, with the central component $\mathrm{C}$ having the largest extinction. Our extinction determination is in rather good agreement with previous results, $A_{V}=7-14$, from $[\mathrm{S} \mathrm{III}] / \mathrm{H} \alpha$ ratio (Young et al. 1988), from $\mathrm{Br} \gamma / \mathrm{H} \alpha$ (Stanford 1991) and from NIR colours (Bushouse \& Werner 1990; Stanford \& Balcells 1990).

\subsubsection{NGC 1614}

The central region of NGC 1614 suffers heavy dust extinction, although less severe than in NGC $520 \mathrm{PN}$. We derive extinction of $A_{K}=0.41$ from recombination line fluxes for the central region of NGC 1614 . Very similar values are derived from the NIR colours, $A_{K}=0.41$ and 0.38 for the SE and NW regions, respectively. These values are in good agreement with previous results, $A_{V}=3-5$, from hydrogen recombination line ratios (Puxley \& Brand 1994; Shier et al. 1996), from $\mathrm{Pa} \beta /[\mathrm{Fe} \mathrm{II}]$ ratio (Puxley et al. 1994) and from NIR colours (Neff et al. 1990; Oliva et al. 1995; Shier et al. 1996).

Puxley \& Brand (1994) determined from NIR spectroscopy that the dust distribution in NGC 1614 is best described either by clumpy foreground dust, implying $A_{V}$ $\sim 4.7$, or by homogeneous foreground dust mixed with internal dust, implying $A_{V} \sim 15$. The fact that the levels of extinction deduced from the line ratios and the NIR colours are very similar indicates that a foreground screen of equal strength for stars and ionized gas is a good approximation for NGC 1614 (see also e.g. Shier et al. 1996).

\subsubsection{NGC 7714}

The central region of NGC 7714 suffers the least amount of extinction of the three galaxies studied, with $A_{K}=0.18$ derived from the recombination line fluxes. Again, very similar values are derived from the NIR colours for the emission regions, $0.13<A_{K}<0.21$ with, interestingly, the strong near-nuclear peak showing the smallest amount of extinction. Thus, the circumnuclear SBs may be more dusty and/or more evolved than the nuclear burst (see also Gonzalez-Delgado et al. 1999). The derived extinction values are in good agreement with previous results, $A_{V}=$ $1-2$, from hydrogen recombination line ratios (Puxley \& Brand 1994) and from NIR colours (Oliva et al. 1995).

\subsection{Star forming properties}

In Table 1 we give for the $\mathrm{Br} \gamma$ emission regions in all galaxies the displacement from the $K$-band nucleus, the aperture diameter, the calibration coefficient from the Airy function, and the observed $\mathrm{Br} \gamma$ and $\mathrm{H}_{2}$ fluxes and $J H K$ colours. In Table 2 we give the extinction derived from the recombination line fluxes and from the $H-K$ colour, and the dereddened $\mathrm{Br} \gamma$ and $\mathrm{H}_{2}$ fluxes, $\mathrm{H}_{2} / \mathrm{Br} \gamma$ ratio and $J H K$ colours for the $\mathrm{Br} \gamma$ regions. The smallest values of FWHM of these regions, $\sim 1$.' $1-1$ ". 4 correspond to a size of the emitting region of $150-250 \mathrm{pc}$. Therefore, the emission regions detected in the NIR actually are conglomerates of several OB associations and giant molecular clouds, probably similar to scaled-up versions of the 30 Dor H II region in the LMC (e.g. Walborn et al. 1999).

The $\mathrm{H}_{2} / \mathrm{Br} \gamma$ ratio can give important clues about the excitation mechanism(s) of the hot molecular gas (Puxley et al. 1990). The main mechanisms suggested are thermal excitation in hot gas by low velocity shocks (e.g. Draine et al. 1983) or by intense X-ray radiation (e.g. Maloney et al. 1996), and fluorescent excitation by strong UV radiation (e.g. Black \& van Dishoeck 1987). The dereddened $\mathrm{H}_{2} / \mathrm{Br} \gamma$ ratios span a range $0.29-0.48$ in NGC 520 PN, 0.14-0.20 in NGC 1614 and 0.12-0.62 in NGC 7714. None of these ratios can be explained by shock excitation caused by cloud-cloud collisions or a $\mathrm{SN}-$ driven wind 
Table 1. Observed $\operatorname{Br} \gamma$ emission regions in the galaxies

\begin{tabular}{lcccccccc}
\hline Region & $\begin{array}{c}\Delta \mathrm{RA} \\
\prime\end{array}$ & $\begin{array}{c}\Delta \text { DEC } \\
\prime\end{array}$ & $\begin{array}{c}\text { Apert } \\
\prime \prime\end{array}$ & $\operatorname{corr}^{a}$ & $\begin{array}{c}\mathrm{Br} \gamma \\
10^{-15} \mathrm{ergs} \mathrm{s}^{-1} \mathrm{~cm}^{-2}\end{array}$ & $\begin{array}{c}J-H \\
\mathrm{mag}\end{array}$ & $\begin{array}{c}H-K \\
\mathrm{mag}\end{array}$ \\
\hline NGC 520 E & 1.6 & 0.1 & 1.4 & 1.11 & 2.14 & 0.965 & 1.79 & 1.19 \\
NGC 520 C & 0.1 & 0.0 & 1.4 & 1.06 & 2.58 & 0.738 & 1.80 & 1.27 \\
NGC 520 W & -1.5 & 0.3 & 1.4 & 1.04 & 1.98 & 0.545 & 1.72 & 1.01 \\
NGC 520 ALL & 0.0 & 0.0 & 5.6 & - & 11.9 & 7.29 & 1.41 & 1.00 \\
NGC 1614 SE & 0.2 & -0.3 & 0.8 & 1.05 & 4.15 & 0.816 & 0.73 & 0.48 \\
NGC 1614 NW & -0.3 & 0.3 & 0.6 & 1.11 & 1.88 & 0.264 & 0.72 & 0.47 \\
NGC 1614 ALL & 0.0 & 0.0 & 3.9 & - & 23.8 & 9.22 & 0.74 & 0.49 \\
NGC 7714 1 & -0.4 & -0.7 & $2.8 \times 1.7$ & 1.04 & 11.9 & 1.47 & 0.63 & 0.30 \\
NGC 7714 2 & 2.6 & -0.6 & 1.7 & 1.01 & 1.16 & 0.443 & 0.65 & 0.34 \\
NGC 7714 3 & 3.4 & -1.7 & 1.1 & 1.01 & 0.346 & 0.211 & 0.67 & 0.36 \\
NGC 7714 4 & 4.5 & -2.7 & 1.7 & 1.01 & 0.897 & 0.171 & 0.64 & 0.34 \\
NGC 7714 N & 0.0 & 0.0 & 3.4 & - & 17.5 & 2.79 & 0.63 & 0.31 \\
NGC 7714 ALL & 0.0 & 0.0 & 11.2 & - & 27.3 & 8.35 & 0.64 & 0.28 \\
\hline
\end{tabular}

${ }^{a}$ Correction factor for the velocity field.

Table 2. Dereddened $\operatorname{Br} \gamma$ emission regions in the galaxies

\begin{tabular}{lccccccc}
\hline Region & $\begin{array}{c}A_{K}^{a} \\
\text { mag }\end{array}$ & $\begin{array}{c}A_{K}^{b} \\
\mathrm{mag}\end{array}$ & $\begin{array}{c}\mathrm{Br} \gamma \\
10^{-15} \mathrm{ergs} \mathrm{s}^{-1} \mathrm{~cm}^{-2}\end{array}$ & $\begin{array}{c}\mathrm{H}_{2} \\
\text { rr }\end{array}$ & $\begin{array}{c}J-H \\
\mathrm{mag}\end{array}$ & $\begin{array}{c}H-K \\
\mathrm{mag}\end{array}$ \\
\hline NGC 520 E & - & 1.50 & 8.86 & 4.23 & 0.477 & 0.13 & 0.22 \\
NGC 520 C & - & 1.62 & 12.0 & 3.64 & 0.303 & 0.00 & 0.22 \\
NGC 520 W & - & 1.21 & 6.22 & 1.79 & 0.288 & 0.38 & 0.22 \\
NGC 520 ALL & - & 1.21 & 37.4 & 24.0 & 0.641 & 0.07 & 0.22 \\
NGC 1614 SE & 0.41 & 0.41 & 6.12 & 1.22 & 0.200 & 0.28 & 0.22 \\
NGC 1614 NW & 0.41 & 0.38 & 2.80 & 0.395 & 0.141 & 0.27 & 0.20 \\
NGC 1614 ALL & 0.41 & 0.41 & 35.2 & 13.8 & 0.392 & 0.28 & 0.22 \\
NGC 7714 1 & 0.18 & 0.13 & 14.1 & 1.76 & 0.125 & 0.43 & 0.19 \\
NGC 7714 2 & 0.18 & 0.19 & 1.38 & 0.529 & 0.384 & 0.45 & 0.22 \\
NGC 7714 3 & 0.18 & 0.21 & 0.410 & 0.252 & 0.615 & 0.47 & 0.24 \\
NGC 7714 4 & 0.18 & 0.18 & 1.06 & 0.204 & 0.192 & 0.44 & 0.22 \\
NGC 7714 N & 0.18 & 0.14 & 20.8 & 3.33 & 0.160 & 0.43 & 0.20 \\
NGC 7714 ALL & 0.18 & 0.10 & 32.4 & 9.96 & 0.308 & 0.44 & 0.17 \\
\hline${ }^{a}$ Determined from literature emission line fluxes, assuming & $A_{\lambda} \propto \lambda^{-1.85}$ (Landini et al. 1984). \\
${ }^{b}$ Estimated from the $H-K$ colour. & & & & &
\end{tabular}

$\left(\mathrm{H}_{2} / \mathrm{Br} \gamma>1\right)$. Interestingly, only two of the $\mathrm{Br} \gamma$ regions (NGC 520 E and NGC 7714 3) can readily be explained with fluorescent UV excitation by individual hot young stars $\left(\mathrm{H}_{2} / \mathrm{Br} \gamma=0.4-0.9\right)$, while for the large majority of them, the line ratios of $<0.4$ are in agreement with fluorescent excitation by intense UV radiation from a large compact cluster of hot stars (Puxley et al. 1990).

For NGC 1614, our line ratios are in good agreement with those derived by Goldader et al. (1997; 0.214 in a $3^{\prime \prime}$ $\times 12^{\prime \prime}$ aperture) and Moorwood \& Oliva (1988, 0.203 in a $6^{\prime \prime}$ aperture). Our ratios for NGC 7714 agree well with that by Taniguchi et al. (1988; 0.172 in a $3 . .5 \times 7^{\prime \prime}$ aperture) and the upper limit by Moorwood \& Oliva $(1988 ;<0.333$ in a $6^{\prime \prime}$ aperture).

The SF properties were interpreted using the comprehensive stellar population synthesis model for galaxies with active SF by Leitherer et al. (1999; hereafter L99). It implements the latest stellar evolutionary tracks (Charbonnel et al. 1999) and atmosphere models (Lejeune et al. 1997), but does not treat self-consistently chemical evolution, binary evolution, mass loss and mixing processes, and late phases of stellar evolution (L99). Despite these shortcomings, it may be considered the most up to date of the suite of models available for studying SF galaxies (see e.g. Leitherer et al. 1996 and references 
therein). In particular, although the exact age of the SB is rather model-dependent, the general conclusion of young vs. old SB remains model-independent (see discussion in Reunanen et al. 2000).

The L99 model predicts the evolution of NIR, optical and UV spectral features as a function of the burst age, metallicity, and the initial mass function (IMF) with lower and upper mass cutoff and slope $\alpha$, for the limiting cases of instantaneous burst of SF (ISF) and constant SF rate (CSFR). The key parameter is the $E W$ of $\operatorname{Br} \gamma$, which is sensitive to the age of the $\mathrm{SB}$ since it measures the ratio of young hot stars $(\mathrm{Br} \gamma)$ and evolved RSGs ( $K$-band continuum) independently of extinction. The estimation of $E W(\operatorname{Br} \gamma)$ was unfortunately complicated by the impossibility to adequately subtract a de Vaucouleurs bulge model (depicting the old stellar population; Kotilainen et al. 2000) from the $K$-band image in these disturbed, peculiar galaxies. The $\operatorname{Br} \gamma E W$ was thus determined by dividing the $\mathrm{Br} \gamma$ fluxes with the total $K$-band fluxes. The resulting $E W$ s are, therefore, lower limits and the derived SB ages upper limits only. However, for strong compact SBs as in these galaxies, a large fraction of the continuum light probably arises from the SB population and therefore the derived lower and upper limits are probably not far from the real values.

The L99 models predict the number of ionizing photons below $912 \AA, N\left(\mathrm{H}^{0}\right)$, which can also be estimated from the $\operatorname{Br} \gamma$ flux. $N\left(\mathrm{H}^{0}\right)$ allows us to evaluate the mass of recently formed stars (in ISF) or the SFR via an assumed IMF (in CSFR). Since we do not have enough data for more detailed modelling, and to allow for an easy comparison with previous results, we assume solar metallicity, $\alpha=2.35$, and consider two models in what follows: (1) ISF with $M_{\mathrm{u}}=100 M_{\odot}$ and (2) CSFR with $M_{\mathrm{u}}=$ $30 M_{\odot}$. The $\mathrm{SF}$ properties $\left(N\left(\mathrm{H}^{0}\right), E W(\mathrm{Br} \gamma)\right.$, age, mass, $\mathrm{SFR}$ and $\nu_{\mathrm{SN}}$ ) of the emission regions in the galaxies were determined by comparing the observed quantities with the L99 models, and are given in Table 3.

The lower limits for the $\operatorname{Br} \gamma E W$ s are small, 11-14

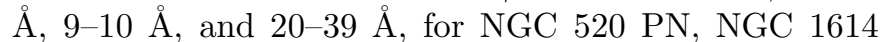
and NGC 7714, respectively. Assuming the CSFR model with $M_{\mathrm{u}}=30 M_{\odot}$, we derive relatively high upper limits for the burst ages, 17-26 Myr, 36-38 Myr, and 9-11 Myr. With the exception of NGC 7714 , these ages get close to the lifetime of individual giant molecular clouds in our Galaxy, after which turbulence and heating from SNe disrupt them and inhibit further SF ( 20-40 Myr; e.g. Blitz 1991). These ages are also in disagreement with the very clumpy NIR morphologies of the galaxies. The corresponding SFR is $0.9-1.8 M_{\odot} \mathrm{yr}^{-1}, 2.1-4.8 M_{\odot} \mathrm{yr}^{-1}$, and $0.1-$ $3.9 M_{\odot} \mathrm{yr}^{-1}$, mass is $18-4710^{6} M_{\odot}, 8-1710^{7} M_{\odot}$, and 1.1-44 $10^{6} M_{\odot}$, and SN rate is $9-2510^{-3} \mathrm{yr}^{-1}, 0.037-$ $0.087 \mathrm{yr}^{-1}$ and $0.4-1910^{-3} \mathrm{yr}^{-1}$ per region for the three galaxies.

Assuming the ISF model, we derive much shorter upper limits for the ages, 6-7 Myr for NGC $520 \mathrm{PN}$ and NGC 1614, and $\sim 6$ Myr for NGC 7714. The corresponding masses of the emission regions are 28-49 $10^{6} M_{\odot}$,
7.5-16 $10^{7} \quad M_{\odot}$, and $2-100 \quad 10^{7} \quad M_{\odot}$, SFR is $4.4-$ $7.5 M_{\odot} \mathrm{yr}^{-1}, 11-25 M_{\odot} \mathrm{yr}^{-1}$, and $0.4-16 M_{\odot} \mathrm{yr}^{-1}$, and the $\mathrm{SN}$ rate is $V_{\mathrm{SN}}=26-4610^{-3} \mathrm{yr}^{-1}, 69-15010^{-3} \mathrm{yr}^{-1}$, and 2.4-97 $10^{-3} \mathrm{yr}^{-1}$, for NGC 520 PN, NGC 1614 and NGC 7714, respectively.

The total mass of the hot gas in the $\operatorname{Br} \gamma$ regions of the galaxies, assuming the ISF model, $\sim 1.210^{8} M_{\odot}$, $\sim 2.410^{8} M_{\odot}$ and $\sim 1.210^{8} M_{\odot}$, although large, is still only a small fraction of the total molecular gas mass, $\sim 4.310^{9} M_{\odot}$ (Yun \& Hibbard 2000), $\sim 1.110^{10} M_{\odot}$ (Sanders et al. 1991) and $\sim 2.110^{9} M_{\odot}$ (Sanders et al. 1991), in NGC 520 PN, NGC 1614 and NGC 7714, respectively.

Stanford (1991) derived from extinction-corrected $\mathrm{H} \alpha$ fluxes $\mathrm{SFR}=0.7 M_{\odot} \mathrm{yr}^{-1}$ for NGC $520 \mathrm{PN}$. The SFR in the PN is much higher than the SFR for a normal isolated spiral galaxy $\left(0.02 M_{\odot} \mathrm{yr}^{-1}\right.$; Keel 1983). On the other hand, the SFR derived from the bolometric luminosity is $7 M_{\odot} \mathrm{yr}^{-1}$ for NGC $520 \mathrm{PN}$ (Stanford 1991). Thus, the $\mathrm{SB}$ in the $\mathrm{PN}$ is unable by an order of magnitude to produce the bolometric luminosity, possibly caused by the old stellar population accounting for some fraction of the luminosity, or because the SB has just ended.

The $E W(\mathrm{Br} \gamma)$, the $E W(\mathrm{CO})$ and the $N(\mathrm{Lyc}) / L(\mathrm{bol})$ ratio all indicate a burst age between 6 and $8 \mathrm{Myr}$ for NGC 1614 (Puxley \& Brand 1999), implying that the most massive stars have already disappeared. The CO spectroscopic index $=0.26$ indicates that about half of the $K-$ band light arises from the old population. For the case of ISF, Puxley \& Brand (1999) derive the mass of formed stars to be $0.3-2510^{9} M_{\odot}$ for different IMFs. The very low $M / L$ ratio of NGC 1614 (0.003; Joseph \& Wright 1985) also excludes a stellar population older than $\sim 16 \mathrm{Myr}$. The conclusion of a short duration $(<1 \mathrm{Myr})$, recent $(<6 \mathrm{Myr})$ burst of SF is strongly supported by the presence of $\mathrm{W}-\mathrm{R}$ features from a population of massive luminous young stars (Vacca \& Conti 1992).

Garcia-Vargas et al. (1997) modeled the NGC 7714 circumnuclear SB regions with a young burst of age 3.55 Myr to explain the emission line spectrum and the detected $\mathrm{W}-\mathrm{R}$ features (GD95). In the optical spectrum of their region A (our region 4) $5^{\prime \prime} \mathrm{SE}$ of the nucleus, they detect Ca II triplet absorption $8600 \AA$ features, compatible with two populations, the ionizing population with age $\sim 5 \mathrm{Myr}$, and a relatively young RSG-rich population of $\sim 10 \mathrm{Myr}$, responsible for the Ca triplet and the Balmer absorption. Further modeling of the UV-NIR spectrum of the nucleus of NGC 7714 (Gonzalez-Delgado et al. 1999) found a best fit for a $\sim 4.5$ Myr burst, with upper mass cutoff $>40 M_{\odot}$. Gonzalez-Delgado et al. (1999) derive a $\mathrm{SN}$ rate $=0.007 \mathrm{yr}^{-1}$ both from the spectral modelling and from radio emission, consistent with the nondetection of SNe in NGC 7714 in the monitoring by Richmond et al. (1998).

The $M / L$ ratio of NGC 7714 is 0.020 (Bernlöhr 1993a), while normal spirals span a range of $0.3<M / L<2.8$. The small $M / L$ ratios found in all three galaxies indicate an IMF biased toward high mass stars, probably through an 
Table 3. Star formation properties of the galaxies

\begin{tabular}{|c|c|c|c|c|c|c|c|c|c|c|}
\hline \multirow[b]{2}{*}{ Region } & \multirow[b]{2}{*}{$N\left(\mathrm{H}^{0}\right)$} & \multirow[b]{2}{*}{$E W(\min )$} & \multicolumn{4}{|c|}{ instantaneous star formation } & \multicolumn{4}{|c|}{ constant star formation rate } \\
\hline & & & age & mass & $\mathrm{SFR}^{a}$ & $v_{\mathrm{SN}}$ & age & $\operatorname{mass}^{b}$ & SFR & $v_{\mathrm{SN}}$ \\
\hline & $10^{52} \mathrm{~s}^{-1}$ & $\AA$ & Myr & $10^{6} M_{\odot}$ & $M_{\odot} \mathrm{yr}^{-1}$ & $10^{-3} \mathrm{yr}^{-1}$ & Myr & $10^{6} M_{\odot}$ & $M_{\odot} \mathrm{yr}^{-1}$ & $10^{-3} \mathrm{yr}^{-1}$ \\
\hline NGC $520 \mathrm{E}$ & 6.03 & 14.1 & 6.46 & 40.3 & 6.23 & 36.8 & 16.6 & 21.8 & 1.31 & 11.1 \\
\hline NGC $520 \mathrm{C}$ & 8.16 & 11.4 & 6.55 & 49.0 & 7.47 & 46.0 & 26.4 & 46.8 & 1.77 & 24.8 \\
\hline NGC $520 \mathrm{~W}$ & 4.23 & 13.2 & 6.47 & 28.5 & 4.40 & 25.9 & 19.4 & 17.8 & 0.92 & 9.38 \\
\hline NGC 520 ALL & 25.5 & 9.20 & 6.61 & 142. & 21.4 & 131. & 40.5 & 162. & 4.01 & 71.6 \\
\hline NGC 1614 SE & 21.7 & 9.72 & 6.59 & 165. & 25.0 & 151. & 36.0 & 171. & 4.75 & 87.0 \\
\hline NGC 1614 NW & 9.96 & 9.45 & 6.60 & 75.4 & 11.4 & 69.5 & 38.4 & 82.6 & 2.15 & 37.5 \\
\hline NGC 1614 ALL & 125. & 9.35 & 6.61 & 949. & 144. & 876. & 39.1 & 1050. & 26.9 & 471. \\
\hline NGC 77141 & 17.8 & 19.9 & 6.36 & 103. & 16.2 & 97.5 & 11.4 & 44.5 & 3.90 & 18.5 \\
\hline NGC 77142 & 1.74 & 22.0 & 6.33 & 9.53 & 1.51 & 9.01 & 10.8 & 4.11 & 0.381 & 1.61 \\
\hline NGC 77143 & 0.518 & 28.0 & 6.25 & 2.47 & 0.40 & 2.37 & 9.82 & 1.12 & 0.114 & 0.39 \\
\hline NGC 77144 & 1.34 & 39.1 & 6.13 & 5.42 & 0.88 & 4.86 & 9.02 & 2.68 & 0.297 & 0.82 \\
\hline NGC 7714 N & 26.3 & 15.3 & 6.43 & 144. & 22.5 & 134. & 14.2 & 68.6 & 4.83 & 33.3 \\
\hline NGC 7714 ALL & 40.9 & 12.6 & 6.50 & 238. & 36.7 & 214. & 21.3 & 160. & 7.50 & 84.3 \\
\hline
\end{tabular}

${ }^{a}$ Mass divided by age.

${ }^{b}$ SFR multiplied by age

increased lower mass cutoff (see also e.g. Rieke et al. 1980; Wright et al. 1988). The low $M / L$ ratios also indicate that the SB has already produced enough RSGs to dominate the NIR emission over the RGB stars from the old population, indicating that the mass of the SB population must be at least a few per cent of the total mass.

\subsection{Gas masses}

Using the average surface brightnesses of the nuclear $\mathrm{H}_{2}$ emission of the galaxies in the largest aperture (see Table 2), assuming $T_{\text {vib }}=2000 \mathrm{~K}$, and following the method of Meaburn et al. (1998), we derive $\sim 1000 M_{\odot}$, $\sim 3000 M_{\odot}$ and $\sim 800 M_{\odot}$ for the mass of the excited nuclear $\mathrm{H}_{2}$ in NGC 520 PN, NGC 1614 and NGC 7714, respectively. These values should be multiplied by an unknown but probably small factor for the linewidth, which may be broader than the width of the F-P passband (see Sect. 2). The resulting masses are similar to those derived for the SB NGC $7771\left(1700 M_{\odot}\right.$; Reunanen et al. 2000) and the SB/Seyfert NGC 3079 (1200 $M_{\odot}$; Meaburn et al. 1998), but much larger than derived for the Seyferts NGC 1097 (120 $M_{\odot}$; Kotilainen et al. 2000), NGC 6574 (80 $M_{\odot}$; Kotilainen et al. 2000) and NGC $3227\left(400 M_{\odot}\right.$; Fernandez et al. 1999). Interestingly, in this small sample of galaxies, the SBs have a much larger $\mathrm{H}_{2}$ mass than the Seyferts. This comparison should be applied to a much larger sample of SBs and Seyferts to verify any difference in the amount of hot molecular material, and to study its implications for the fuelling of nuclear activity.

\section{Conclusions}

We present high spatial resolution $(\sim 0$ "' 6$)$ near-infrared broad-band $J H K$ images and $\operatorname{Br} \gamma 2.1661 \mu \mathrm{m}$ and $\mathrm{H}_{2} 1-0$ $S(1) 2.122 \mu \mathrm{m}$ emission line images of the nuclear regions in the interacting starburst galaxies NGC 520, NGC 1614 and NGC 7714. The near-infrared emission line and radio morphologies are in general agreement, although there are differences in details. In NGC 1614, we detect a nuclear double structure in $\operatorname{Br} \gamma$, in agreement with the radio double structure. We derive average extinctions of $A_{K}=0.41$ and $A_{K}=0.18$ toward the nuclear regions of NGC 1614 and NGC 7714, respectively. For NGC 520, the extinction is much higher, $A_{K}=1.2-1.6$. The observed $\mathrm{H}_{2} / \mathrm{Br} \gamma$ ratios indicate that the main excitation mechanism of the molecular gas is fluorescence by intense UV radiation from clusters of hot young stars, while shock excitation can be ruled out.

The starburst regions in all galaxies exhibit small $\operatorname{Br} \gamma$ equivalent widths. Assuming a constant star formation model, even with a lowered upper mass cutoff of $M_{\mathrm{u}}$ $=30 M_{\odot}$, results in rather old ages (10-40 Myr), in disagreement with the clumpy near-infrared morphologies. We prefer a model of an instantaneous burst of star formation with $M_{\mathrm{u}}=100 M_{\odot}$ occurring $\sim 6-7 \mathrm{Myr}$ ago, in agreement with previous determinations and with the detection of W-R features in NGC 1614 and NGC 7714. Finally, we note a possible systematic difference in the amount of hot molecular gas between starburst and Seyfert galaxies.

Acknowledgements. The United Kingdom Infrared Telescope is operated by the Joint Astronomy Centre on behalf of the UK Particle Physics and Astronomy Research Council. Thanks are due to Tom Geballe and Thor Wold for assistance during the observations, and to the anonymous referee for comments that helped to clarify the presentation. This research has made use of the NASA/IPAC Extragalactic Database (NED), which is operated by the Jet Propulsion Laboratory, California Institute of Technology, under contract with the National Aeronautics and Space Administration. 


\section{References}

Aitken, D. K., Roche, P. F., \& Phillips, M. M. 1981, MNRAS, 196, 101P

Armus, L., Heckman, T. M., \& Miley, G. K. 1989, ApJ, 347, 727

Barnes, J. E., \& Hernquist, L. E. 1991, ApJ, 370, L65

Bernlöhr, K. 1993a, A\&A, 268, 25

Bernlöhr, K. 1993b, A\&A, 270, 20

Black, J. H., \& van Dishoeck, E. F. 1987, ApJ, 322, 412

Bland-Hawthorn, J. 1995, Tridimensional Optical Spectroscopic Methods in Astrophysics, ed. G. Comte, \& M. Marcelin, ASP Conf. Ser., 71, 72

Bushouse, H. A., \& Werner, M. W. 1990, ApJ, 359, 72

Calzetti, D. 1997, AJ, 113, 162

Calzetti, D., Kinney, A. L., \& Storchi-Bergmann, T. 1996, ApJ, 458,132

Carico, D. P., Graham, J. R., Matthews, K., et al. 1990, ApJ, 349, L39

Carral, P., Turner, J. L., \& Ho, P. T. P. 1991, ApJ, 362, 434

Chapelon, S., Contini, T., Davoust, E. 1999, A\&A, 345, 81

Charbonnel, C., Däppen, W., Schaerer, D., et al. 1999, A\&AS, 135,405

Condon, J. J., Condon, M. A., Gisler, G., \& Puschell, J. J. 1982, ApJ, 252, 102

Condon, J. J., Helou, G., Sanders, D. B., \& Soifer, B. T. 1990, ApJS, 73, 359

De Robertis, M. M., \& Shaw, R. A. 1988, ApJ, 329, 629

Draine, B. T., Roberge, W. G., \& Dalgarno, A. 1983, ApJ, 264, 485

Fernandez, B. R., Holloway, A. J., Meaburn, J., Pedlar, A., \& Mundell, C. G. 1999, MNRAS, 305, 319

Forbes, D. A., Ward, M. J., DePoy, D. L., Boisson, C., \& Smith, M. S. 1992, MNRAS, 254, 509

Garcia-Vargas, M. L., Gonzalez-Delgado, R. M., Perez, E., et al. 1997, ApJ, 478, 112

Glass, I. S., \& Moorwood, A. F. M. 1985, MNRAS, 214, 429

Goldader, J. D., Joseph, R. D., Doyon, R., \& Sanders, D. B. 1997, ApJ, 474, 104

Gonzalez-Delgado, R. M., Perez, E., Diaz, A., et al. 1995, ApJ, 439, 604 (GD95)

Gonzalez-Delgado, R. M., Garcia-Vargas, M. L., Goldader, J., Leitherer, C., \& Pasquali, A., 1999, ApJ, 513, 707

Graham, J. R., Carico, D. P., Matthews, K., et al. 1990, ApJ, $354, \mathrm{~L} 5$

Hibbard, J. E., \& van Gorkom, J. H. 1996, AJ, 111, 655

Ho, P. T. P., Beck, S. C., \& Turner, J. L. 1990, ApJ, 349, 57

Joseph, R. D., \& Wright, G. S. 1985, MNRAS, 214, 87

Keel, W. C. 1983, ApJ, 269, 466

Keto, E., Ball, R., Arens, J., Jernigan, \& G., Meixner, M. 1992, ApJ, 389, 223

Kotilainen, J. K., Reunanen, J., Laine, S., \& Ryder, S. D. 2000, A\&A, 353, 834

Landini, M., Natta, A., Salinari, P., Oliva, E., \& Moorwood, A. F. M. 1984, A\&A, 134, 284
Leitherer, C., et al. 1996, PASP, 108, 996

Leitherer, C., Schaerer, D., Goldader, J. D., et al. 1999, ApJS, 123, 3 (L99)

Lejeune, T., Buser, R., \& Cuisinier, F. 1997, A\&AS, 125, 229

Maloney, P. R., Hollenbach, D. J., \& Tielens, A. G. G. M. 1996, ApJ, 466, 561

Meaburn, J., Fernandez, B. R., Holloway, A. J., et al. 1998, MNRAS, 295, L45

Mezger, P. G., Duschl, W. J., \& Zylka, R. 1996, A\&AR, 7, 289

Moorwood, A. F. M, \& Oliva, E. 1988, A\&A, 203, 278

Neff, S. G., Hutchings, J. B., Stanford, S. A., \& Unger, S. W. 1990, AJ, 99, 1088

Norman, C. A., Bowen, D. V., Heckman, T., Blades, C., \& Danly, L. 1996, ApJ, 472, 73

Oliva, E., Origlia, L., Kotilainen, J. K., \& Moorwood, A. F. M. 1995, A\&A, 301, 55

Papaderos, P., \& Fricke, K. J. 1998, A\&A, 338, 31

Puxley, P. J., \& Brand, P. W. J. L. 1994, MNRAS, 266, 431

Puxley, P. J., \& Brand, P. W. J. L. 1999, ApJ, 514, 675

Puxley, P. J., Hawarden, T. G., \& Mountain, C. M. 1990, ApJ, 364,77

Puxley, P. J., Lumsden, S. L., Brand, P. W. J. L., \& Doyon, R. 1994, MNRAS, 270, L7

Reunanen, J., Kotilainen, J. K., Laine, S., \& Ryder, S. D. 2000, ApJ, 529, 853

Richmond, M. W., Filippenko, A. V., \& Galisky, J. 1998, PASP, 110,553

Rieke, G. H., Lebofsky, M. J., Thompson, R. J., Low, F. J., \& Tokunaga, A. T. 1980, ApJ, 238, 24

Rieke, G. H., Loken, K., Rieke, M. J., \& Tamblyn, P. 1993, ApJ, 412, 99

Rownd, B. K., \& Young, J. S. 1999, AJ, 118, 670

Sanders, D. B., Scoville, N. Z., \& Soifer, B. T. 1991, ApJ, 370, 158

Scoville, N. Z., Sanders, D. B., Sargent, A. I., Soifer, B. T., \& Tinney, C. G. 1989, ApJ, 345, L25

Shier, L. M., Rieke, M. J., \& Rieke, G. H. 1996, ApJ, 470, 222

Smith, B. J., Struck, C., \& Pogge, R. W. 1997, ApJ, 483, 754

Stanford, S. A. 1991, ApJ, 381, 409

Stanford, S. A., \& Balcells, M. 1990, ApJ, 355, 59

Stanford, S. A., \& Balcells, M. 1991, ApJ, 370, 118

Storchi-Bergmann, T., Kinney, A. L., \& Challis, P. 1995, ApJS, 98, 103

Taniguchi, Y., Kawara, K., Nishida, M., Tamura, S., \& Nishida, M. T. 1988, AJ, 95, 1378

Telesco, C. M., Wolstencroft, R. D., \& Done, C. 1988, ApJ, 329,174

Vacca, W. D., \& Conti, P. S. 1992, ApJ, 401, 543

Walborn, N. R., Barba, R. H., Brandner, W., et al. 1999, AJ, 117,225

Wright, G. S., Joseph, R. D., Robertson, N. A., James, P. A., \& Meikle, W. P. S. 1988, MNRAS, 233, 1

Young, J. S., Kleinmann, S. G., \& Allen, L. E. 1988, ApJ, 334, L63

Yun, M. S., \& Hibbard, J. E. 2000, ApJ, in press 